\title{
On counterexamples to a conjecture of Wills and Ehrhart polynomials whose roots have equal real parts
}

\author{
Matthias Henze* \\ Institut für Informatik \\ Freie Universität Berlin \\ Berlin, Germany \\ matthias.henze@fu-berlin.de
}

Submitted: Sep 26, 2013; Accepted: Jan 17, 2014; Published: Feb 7, 2014

Mathematics Subject Classifications: 52B20, 11H06, 52A40

\begin{abstract}
As a discrete analog to Minkowski's theorem on convex bodies, Wills conjectured that the Ehrhart coefficients of a 0-symmetric lattice polytope with exactly one interior lattice point are maximized by those of the cube of side length two. We discuss several counterexamples to this conjecture and, on the positive side, we identify a family of lattice polytopes that fulfill the claimed inequalities. This family is related to the recently introduced class of $l$-reflexive polytopes.
\end{abstract}

Keywords: Wills' conjecture, $l$-reflexive polytope, Ehrhart polynomial

\section{Introduction}

Let $\mathcal{P}^{n}$ denote the family of all lattice polytopes in $\mathbb{R}^{n}$, that is, the convex hulls of finitely many points from the integer lattice $\mathbb{Z}^{n}$. Such a polytope $P \in \mathcal{P}^{n}$ is called 0 -symmetric if $P=-P$. Its volume (Lebesgue measure) is denoted by $\operatorname{vol}(P)$ and its discrete volume, the lattice point enumerator, by $\mathrm{G}(P)=\#\left(P \cap \mathbb{Z}^{n}\right)$.

Minkowski [17] proved that among the 0-symmetric compact convex sets that have only the origin as an interior lattice point, the cube $C_{n}=[-1,1]^{n}$ has the biggest volume and contains the most lattice points. More precisely, for every such set $K \subset \mathbb{R}^{n}$,

$$
\operatorname{vol}(K) \leqslant \operatorname{vol}\left(C_{n}\right)=2^{n} \quad \text { and } \quad \mathrm{G}(K) \leqslant \mathrm{G}\left(C_{n}\right)=3^{n} .
$$

*Partially supported by the Deutsche Forschungsgemeinschaft (DFG) within the project He 2272/4-1 and by the ESF EUROCORES programme EuroGIGA-VORONOI, (DFG): Ro 2338/5-1. 
Wills [19] proposed to further discretize these inequalities for the class of lattice polytopes. A famous result of Ehrhart [10] shows that the counting function $k \mapsto \mathrm{G}(k P)$, for $k \in \mathbb{N}$ a positive integer, is a polynomial in $k$ of degree $n$, whenever $P \in \mathcal{P}^{n}$ is a lattice polytope. This polynomial $\mathrm{G}(k P)=\sum_{i=0}^{n} \mathrm{~g}_{i}(P) k^{i}$ is called the Ehrhart polynomial of $P$ and the numbers $\mathrm{g}_{i}(P)$ are the Ehrhart coefficients. It is not hard to see that $\mathrm{g}_{0}(P)=1$ and $\mathrm{g}_{n}(P)=\operatorname{vol}(P)$. Moreover, also the second highest Ehrhart coefficient $\mathrm{g}_{n-1}$ has a nice geometric meaning which is given in detail in (3.3). For details on Ehrhart theory including extensive references we refer the interested reader to [2]. Now, using the description for $g_{n-1}(P)$, Wills proved that for every 0 -symmetric $P \in \mathcal{P}^{n}$ that has only the origin as an interior lattice point

$$
\mathrm{g}_{n-1}(P) \leqslant \mathrm{g}_{n-1}\left(C_{n}\right)=n 2^{n-1} .
$$

Furthermore, he wondered about a much stronger extremality property of the cube $C_{n}$.

Conjecture 1.1 (Wills' conjecture $[19,12]$ ). Let $P \in \mathcal{P}^{n}$ be a 0 -symmetric lattice polytope with the origin being its only interior lattice point. Then

$$
\mathrm{g}_{i}(P) \leqslant \mathrm{g}_{i}\left(C_{n}\right)=2^{i}\left(\begin{array}{c}
n \\
i
\end{array}\right) \quad \text { for all } i=0, \ldots, n .
$$

Minkowski's inequalities (1.1) nicely embed into these proposed relations, as $\operatorname{vol}(P)=$ $\mathrm{g}_{n}(P)$ and $\mathrm{G}(P)=\sum_{i=0}^{n} \mathrm{~g}_{i}(P)$. Wills proved his conjecture for $n=3$, and it gets further support by the fact that the Ehrhart polynomial of every $P \in \mathcal{P}^{n}$, that meets the conditions above, is pointwise maximized by that one of the cube. That is, $\mathrm{G}(k P) \leqslant$ $\mathrm{G}\left(k C_{n}\right)$, for $k \in \mathbb{N}$, which follows from a discrete version of Minkowski's 1st Theorem due to Betke, Henk \& Wills [3, Thm. 2.1].

Nevertheless, the objective of this paper is to exhibit counterexamples to Wills' Conjecture. On the positive side, we identify a family of lattice polytopes that fulfill the claimed inequalities (Corollary 3.3). This family is related to the recently introduced $l$-reflexive polytopes which we also briefly discuss.

\section{Counterexamples to Wills' conjecture}

In this section, we prove the following theorem which sums up our negative findings in regard of Wills' conjecture. The second part shows that in dimensions $n=4 k+1$ the proposed bound $\mathrm{g}_{1}(P) \leqslant 2 n$ for the first Ehrhart coefficient fails even badly. Recall that the Landau notation $g(n) \in \Theta(f(n))$ means that there are constants $c_{1}, c_{2}>0$ such that $c_{1} f(n) \leqslant g(n) \leqslant c_{2} f(n)$, for all large enough $n \in \mathbb{N}$.

\section{Theorem 2.1.}

i) For every $n \geqslant 7$, there is a 0 -symmetric lattice polytope $P \in \mathcal{P}^{n}$ with int $P \cap \mathbb{Z}^{n}=\{0\}$ that violates Wills' conjecture. In particular, $\mathrm{g}_{1}(P)>\mathrm{g}_{1}\left(C_{n}\right)$. 
ii) For $n \in \mathbb{N}$, let $Q_{n}=\operatorname{conv}\left\{C_{n-1} \times\{0\}, \pm e_{n}\right\}$. Then

$$
\mathrm{g}_{1}\left(Q_{n}\right)=2(n-1)+\left(4-2^{n}\right) B_{n-1},
$$

where $B_{j}$ denotes the $j$ th Bernoulli number. In particular, we have

$$
\mathrm{g}_{1}\left(Q_{n}\right)=2(n-1) \quad \text { for even } n \in \mathbb{N},
$$

and

$$
(-1)^{\frac{n-1}{2}} \mathrm{~g}_{1}\left(Q_{n}\right) \in \Theta\left(\left(\frac{n}{\pi e}\right)^{n}\right) \quad \text { for odd } n \in \mathbb{N} \text {. }
$$

Let $C_{n}^{\star}=\operatorname{conv}\left\{ \pm e_{1}, \ldots, \pm e_{n}\right\}$ be the standard crosspolytope in $\mathcal{P}^{n}$. In order to show part i) of Theorem 2.1, we consider the polytope $P_{n}=\operatorname{conv}\left\{C_{n-1} \times\{0\}, C_{n-1}^{\star} \times\{-1,1\}\right\}$ that arises as the convex hull of an $(n-1)$-dimensional cube put at height 0 and two $(n-1)$ dimensional crosspolytopes put at height -1 and 1 , respectively. Using polymake [11] and LattE [8], we see that $P_{7}$ is a counterexample to Conjecture 1.1. More precisely, its Ehrhart polynomial is given by

$$
1+\frac{1534}{105} k+\frac{3188}{45} k^{2}+\frac{7112}{45} k^{3}+\frac{1756}{9} k^{4}+\frac{7004}{45} k^{5}+\frac{4952}{45} k^{6}+\frac{15656}{315} k^{7},
$$

and therefore $\mathrm{g}_{1}\left(P_{7}\right)=\frac{1534}{105}>14=\mathrm{g}_{1}\left(C_{7}\right)$.

Remark 2.2. In dimensions 4,5 and 6 we tested Wills' conjecture on lattice polytopes that have a similar structure to $P_{n}$. In particular, we checked all 0-symmetric polytopes that are given as the convex hull of lower dimensional cubes or crosspolytopes put at height 0, -1 and 1. Moreover, we investigated all Hanner polytopes (Ehrhart polynomials can be recursively computed with the help of [6]) in these dimensions, i.e., polytopes arising by successive prism or bipyramid operations (see [14]). None of these examples turned out to be a counterexample.

Now, we want to construct counterexamples in every dimension $n \geqslant 7$, based on the example $P_{7}$ and on an easy formula for Ehrhart coefficients of products of lattice polytopes. For the sake of completeness, we provide the short argument (see also [2, Exs. 2.4]).

Proposition 2.3. Let $P \in \mathcal{P}^{p}$ and $Q \in \mathcal{P}^{q}$ be lattice polytopes. Then

$$
\mathrm{g}_{j}(P \times Q)=\sum_{i=0}^{j} \mathrm{~g}_{i}(P) \mathrm{g}_{j-i}(Q) \quad \text { for all } j=0, \ldots, p+q,
$$

where $\mathrm{g}_{k}(P)=\mathrm{g}_{l}(Q)=0$, for all $k>p$ and $l>q$.

Proof. For every $k \in \mathbb{N}$, we have

$$
\begin{aligned}
\mathrm{G}(k(P \times Q)) & =\mathrm{G}(k P \times k Q)=\mathrm{G}(k P) \mathrm{G}(k Q) \\
& =\left(\sum_{i=0}^{p} \mathrm{~g}_{i}(P) k^{i}\right)\left(\sum_{j=0}^{q} \mathrm{~g}_{j}(Q) k^{j}\right)=\sum_{j=0}^{p+q}\left(\sum_{i=0}^{j} \mathrm{~g}_{i}(P) \mathrm{g}_{j-i}(Q)\right) k^{j} .
\end{aligned}
$$

Comparing coefficients gives the claimed identities. 
The identity in the case $j=1$ implies

$$
\mathrm{g}_{1}\left(P_{7} \times C_{m}\right)=\mathrm{g}_{1}\left(P_{7}\right)+\mathrm{g}_{1}\left(C_{m}\right)>\mathrm{g}_{1}\left(C_{7}\right)+\mathrm{g}_{1}\left(C_{m}\right)=\mathrm{g}_{1}\left(C_{m+7}\right),
$$

for all $m \in \mathbb{N}_{0}$. Hence, we found a counterexample for every dimension $n \geqslant 7$.

Proof of Theorem 2.1 ii). Let $k \in \mathbb{N}$ and let $H_{j}=\left\{x \in \mathbb{R}^{n}: x_{n}=j\right\}$ be the orthogonal plane to $e_{n}$ of height $j$. For $j=0,1, \ldots, k$, the plane $H_{j}$ intersects $k Q_{n}$ in a copy of $C_{n-1}$ scaled by $k-j$. Counting the lattice points in $k Q_{n}$ with respect to these intersections, we find that the Ehrhart polynomial of $Q_{n}$ is given by

$$
\begin{aligned}
\mathrm{G}\left(k Q_{n}\right) & =(2 k+1)^{n-1}+2 \sum_{j=0}^{k-1}(2 j+1)^{n-1} \\
& =(2 k+1)^{n-1}+2 \sum_{j=0}^{k-1} \sum_{i=0}^{n-1}\left(\begin{array}{c}
n-1 \\
i
\end{array}\right)(2 j)^{i} \\
& =\sum_{i=0}^{n-1}\left(\begin{array}{c}
n-1 \\
i
\end{array}\right) 2^{i} k^{i}+2 \sum_{i=0}^{n-1}\left(\begin{array}{c}
n-1 \\
i
\end{array}\right) 2^{i}\left(\sum_{j=0}^{k-1} j^{i}\right) .
\end{aligned}
$$

Faulhaber's formula (see $[1, \S 23.1]$ ) expresses the $\operatorname{sum} \sum_{j=0}^{k-1} j^{i}$ as a polynomial in $k$, more precisely

$$
\sum_{j=0}^{k-1} j^{i}=\frac{1}{i+1} \sum_{j=0}^{i}\left(\begin{array}{c}
i+1 \\
j
\end{array}\right) B_{j} k^{i-j+1}=\frac{1}{i+1} \sum_{j=1}^{i+1}\left(\begin{array}{c}
i+1 \\
j
\end{array}\right) B_{i-j+1} k^{j},
$$

where $B_{j}$ is the $j$ th Bernoulli number. Therefore, we continue as

$$
\begin{aligned}
\mathrm{G}\left(k Q_{n}\right) & =\sum_{i=0}^{n-1}\left(\begin{array}{c}
n-1 \\
i
\end{array}\right) 2^{i} k^{i}+2 \sum_{i=0}^{n-1}\left(\begin{array}{c}
n-1 \\
i
\end{array}\right) \frac{2^{i}}{i+1} \sum_{j=1}^{i+1}\left(\begin{array}{c}
i+1 \\
j
\end{array}\right) B_{i-j+1} k^{j} \\
& =\sum_{i=0}^{n-1}\left(\begin{array}{c}
n-1 \\
i
\end{array}\right) 2^{i} k^{i}+\frac{2}{n} \sum_{j=1}^{n} \sum_{i=j-1}^{n-1}\left(\begin{array}{c}
n \\
i+1
\end{array}\right) 2^{i}\left(\begin{array}{c}
i+1 \\
j
\end{array}\right) B_{i-j+1} k^{j} .
\end{aligned}
$$

Thus, the Ehrhart coefficients of $Q_{n}$ are given by

$$
\mathrm{g}_{i}\left(Q_{n}\right)=\left(\begin{array}{c}
n-1 \\
i
\end{array}\right) 2^{i}+\frac{2}{n} \sum_{j=i-1}^{n-1}\left(\begin{array}{c}
n \\
j+1
\end{array}\right) 2^{j}\left(\begin{array}{c}
j+1 \\
i
\end{array}\right) B_{j-i+1},
$$

for all $i=1, \ldots, n$. In the case $i=1$, this gives us

$$
\mathrm{g}_{1}\left(Q_{n}\right)=2(n-1)+2 \sum_{j=0}^{n-1}\left(\begin{array}{c}
n-1 \\
j
\end{array}\right) 2^{j} B_{j}=2(n-1)+2^{n} B_{n-1}\left(\frac{1}{2}\right),
$$


where $B_{n}(x)=\sum_{j=0}^{n}\left(\begin{array}{c}n \\ j\end{array}\right) x^{n-j} B_{j}$ denotes the $n$th Bernoulli polynomial. In $[1, \S 23.1]$, we find the identity $B_{n}\left(\frac{1}{2}\right)=-\left(1-2^{1-n}\right) B_{n}$, which leads to our desired expression $\mathrm{g}_{1}\left(Q_{n}\right)=2(n-1)+\left(4-2^{n}\right) B_{n-1}$.

Since $B_{j}=0$ for all odd indices $j \geqslant 3$, we have $\mathrm{g}_{1}\left(Q_{n}\right)=2(n-1)$ for all even $n \in \mathbb{N}$. The Bernoulli numbers with even indices satisfy (see $[1, \S 23.1]$ )

$$
\frac{2(2 j) !}{(2 \pi)^{2 j}}<(-1)^{j+1} B_{2 j}<\frac{2(2 j) !}{(2 \pi)^{2 j}}\left(\frac{1}{1-2^{1-2 j}}\right) .
$$

Assuming that $n=2 k+1$ for some $k \in \mathbb{N}$, we obtain

$$
\begin{aligned}
(-1)^{k} \mathrm{~g}_{1}\left(Q_{2 k+1}\right) & =(-1)^{k} 4 k+(-1)^{k}\left(4-2^{2 k+1}\right) B_{2 k} \\
& \in \Theta\left(\frac{2^{2 k+2}(2 k) !}{(2 \pi)^{2 k}}\right)=\Theta\left(\left(\frac{n}{\pi e}\right)^{n}\right) .
\end{aligned}
$$

The last equality comes from Stirling's approximation of the factorial.

The polytopes $Q_{n}=\operatorname{conv}\left\{C_{n-1} \times\{0\}, \pm e_{n}\right\}$ from Theorem 2.1 ii) appeared already in [4, Prop. 1.1] as counterexamples to another conjectured relation between Ehrhart coefficients of 0 -symmetric lattice polytopes. Invoking polymake and LattE again, we find that they moreover serve as counterexamples for Wills' conjecture on higher Ehrhart coefficients. Indeed, we have

$$
\begin{gathered}
\mathrm{g}_{1}\left(Q_{9}\right)=\frac{494}{15}>18=\mathrm{g}_{1}\left(C_{9}\right), \\
\mathrm{g}_{3}\left(Q_{11}\right)=1976>1320=\mathrm{g}_{3}\left(C_{11}\right) \text { and } \\
\mathrm{g}_{5}\left(Q_{13}\right)=\frac{260832}{5}>41184=\mathrm{g}_{5}\left(C_{13}\right) .
\end{gathered}
$$

\section{Lattice polytopes with Ehrhart polynomials with roots of equal real part}

This section deals with linear inequalities in the spirit of Wills' conjecture for a special class of lattice polytopes. The Ehrhart polynomial of a lattice polytope $P \in \mathcal{P}^{n}$ may be understood as a polynomial in a complex variable and thus it makes sense to speak about its roots. Following [5], we denote these roots by $-\gamma_{1}(P), \ldots,-\gamma_{n}(P)$. Using $\mathrm{g}_{n}(P)=\operatorname{vol}(P)$, we get

$$
\mathrm{G}(s P)=\sum_{i=0}^{n} \mathrm{~g}_{i}(P) s^{i}=\operatorname{vol}(P) \prod_{i=1}^{n}\left(s+\gamma_{i}(P)\right) \quad \text { for } \quad s \in \mathbb{C} .
$$

Braun [7] proved that the roots of an Ehrhart polynomial lie in the disc with center $-\frac{1}{2}$ and radius $n\left(n-\frac{1}{2}\right)$. This is only one reason to study the situation in which the real part of all roots equals $-\frac{1}{2}$ as it was done for example in $[2,5]$. The cube $C_{n}$ and the 
crosspolytope $C_{n}^{\star}$ are standard examples of lattice polytopes that belong to this class (see the references above). Our main result here is concerned with a broader class of lattice polytopes.

Theorem 3.1. Let $P \in \mathcal{P}^{n}$ be a lattice polytope with the property that all the roots of its Ehrhart polynomial have real part $-\frac{1}{a}$, for some $a>0$.

i) For all $0 \leqslant s<t \leqslant n$, we have

$$
\frac{\mathrm{g}_{t}(P)}{\mathrm{g}_{s}(P)} \leqslant a^{t-s} \frac{\left(\begin{array}{l}
n \\
t
\end{array}\right)}{\left(\begin{array}{l}
n \\
s
\end{array}\right)} .
$$

For $(s, t)=(n-1, n)$ we have equality. For every $0 \leqslant s<t \leqslant n$ with $(s, t) \neq(n-1, n)$ equality holds if and only if $\mathrm{G}(k P)=(a k+1)^{n}$, for all $k \in \mathbb{N}$.

ii) We have

$$
\operatorname{vol}(P) \leqslant\left(\frac{a}{a+1}\right)^{n} \mathrm{G}(P),
$$

and equality holds if and only if $\mathrm{G}(k P)=(a k+1)^{n}$, for all $k \in \mathbb{N}$.

iii) We have

$$
\mathrm{G}(P) \leqslant \frac{(a+1)^{n-2}(a+2)}{a^{n-1}} \operatorname{vol}(P)+(a+1)^{n-2} .
$$

Equality holds if and only if there is at most one pair of complex conjugate roots with nonzero imaginary part. In particular, equality holds for $n \in\{2,3\}$.

Proof. i): As above, we write

$$
\mathrm{G}(k P)=\operatorname{vol}(P) \prod_{i=1}^{n}\left(k+\gamma_{i}(P)\right) .
$$

By assumption, the real part of $-\gamma_{i}(P)$ equals $-\frac{1}{a}$, for all $i=1, \ldots, n$.

We consider the case $n=2 l$ first. There are $b_{1}, \ldots, b_{l} \in \mathbb{R}$ such that

$$
\begin{aligned}
\frac{\mathrm{G}(k P)}{\operatorname{vol}(P)} & =\prod_{j=1}^{l}\left(k+\frac{1}{a} \pm b_{j} \mathrm{i}\right)=\prod_{j=1}^{l}\left(\left(k+\frac{1}{a}\right)^{2}+b_{j}^{2}\right) \\
& =\sum_{j=0}^{l} \sigma_{l-j}\left(b_{1}^{2}, \ldots, b_{l}^{2}\right)\left(k+\frac{1}{a}\right)^{2 j} \\
& =\sum_{j=0}^{l} \sigma_{l-j}\left(b_{1}^{2}, \ldots, b_{l}^{2}\right) \sum_{t=0}^{2 j}\left(\begin{array}{c}
2 j \\
t
\end{array}\right)\left(\frac{1}{a}\right)^{2 j-t} k^{t} \\
& =\sum_{t=0}^{2 l} a^{t}\left(\sum_{j=\left\lceil\frac{t}{2}\right\rceil}^{l} a^{-2 j} \sigma_{l-j}\left(b_{1}^{2}, \ldots, b_{l}^{2}\right)\left(\begin{array}{c}
2 j \\
t
\end{array}\right)\right) k^{t} .
\end{aligned}
$$


As usual, $\sigma_{j}\left(x_{1}, \ldots, x_{l}\right)=\sum_{1 \leqslant i_{1}<\ldots<i_{j} \leqslant l} \prod_{t=1}^{j} x_{i_{t}}$ denotes the $j$ th elementary symmetric polynomial. From the above identity we can read off a formula for the Ehrhart coefficients $\mathrm{g}_{t}(P)$ and it follows that, for $0 \leqslant s \leqslant t \leqslant n$, the inequality

$$
\frac{\mathrm{g}_{t}(P)}{\mathrm{g}_{s}(P)}=a^{t-s} \frac{\sum_{j=\left\lceil\frac{t}{2}\right\rceil}^{l} a^{-2 j} \sigma_{l-j}\left(b_{1}^{2}, \ldots, b_{l}^{2}\right)\left(\begin{array}{c}
2 j \\
t
\end{array}\right)}{\sum_{j=\left\lceil\frac{s}{2}\right\rceil}^{l} a^{-2 j} \sigma_{l-j}\left(b_{1}^{2}, \ldots, b_{l}^{2}\right)\left(\begin{array}{c}
(j j \\
s
\end{array}\right)} \leqslant a^{t-s} \frac{\left(\begin{array}{c}
n \\
t
\end{array}\right)}{\left(\begin{array}{c}
n \\
s
\end{array}\right)}
$$

is equivalent to

$$
\begin{aligned}
\sum_{j=\left\lceil\frac{t}{2}\right\rceil}^{l} a^{-2 j} \sigma_{l-j} & \left(b_{1}^{2}, \ldots, b_{l}^{2}\right)\left(\begin{array}{c}
2 j \\
t
\end{array}\right)\left(\begin{array}{c}
2 l \\
s
\end{array}\right) \\
& \leqslant \sum_{j=\left\lceil\frac{s}{2}\right\rceil}^{l} a^{-2 j} \sigma_{l-j}\left(b_{1}^{2}, \ldots, b_{l}^{2}\right)\left(\begin{array}{c}
2 j \\
s
\end{array}\right)\left(\begin{array}{c}
2 l \\
t
\end{array}\right) .
\end{aligned}
$$

Since all summands are nonnegative and $t \geqslant s$, it suffices to show that $\left(\begin{array}{c}2 j \\ t\end{array}\right)\left(\begin{array}{c}2 l \\ s\end{array}\right) \leqslant\left(\begin{array}{c}2 j \\ s\end{array}\right)\left(\begin{array}{c}2 l \\ t\end{array}\right)$, for all $j=\left\lceil\frac{t}{2}\right\rceil, \ldots, l$. As this is equivalent to $(2 j-t+1) \cdot \ldots \cdot(2 j-s) \leqslant(2 l-t+1) \cdot \ldots \cdot(2 l-s)$, we are done.

Since $t \geqslant s$, we have the same number of summands on either side of (3.2) if and only if $t$ is even and $s=t-1$. Moreover, since $s \neq t$, we have $\left(\begin{array}{c}2 j \\ t\end{array}\right)\left(\begin{array}{c}2 l \\ s\end{array}\right) \leqslant\left(\begin{array}{c}2 j \\ s\end{array}\right)\left(\begin{array}{c}2 l \\ t\end{array}\right)$ if and only if $j=l$. These two observations imply that we have equality in (3.2) for $(s, t)=(2 l-1,2 l)=(n-1, n)$, and for every other pair $(s, t)$ with $0 \leqslant s<t \leqslant n$ if and only if $b_{1}=\ldots=b_{l}=0$, which is equivalent to $\mathrm{G}(k P)=(a k+1)^{n}$, for all $k \in \mathbb{N}$. Note, that it is not clear that this means that $P$ is unimodularly equivalent to $\frac{a}{2} C_{n}$ (for related work see [13]).

The case of odd dimensions $n=2 l+1$ is similar. In comparison to the even-dimensional case, there is an additional real zero $-\frac{1}{a}$ and we get

$$
\begin{aligned}
\frac{\mathrm{G}(k P)}{\operatorname{vol}(P)}= & \left(k+\frac{1}{a}\right) \prod_{j=1}^{l}\left(k+\frac{1}{a} \pm b_{j} \mathrm{i}\right) \\
= & \left(k+\frac{1}{a}\right) \sum_{t=0}^{2 l} a^{t}\left(\sum_{j=\left\lceil\frac{t}{2}\right\rceil}^{l} a^{-2 j} \sigma_{l-j}\left(b_{1}^{2}, \ldots, b_{l}^{2}\right)\left(\begin{array}{c}
2 j \\
t
\end{array}\right)\right) k^{t} \\
= & \sum_{t=1}^{2 l+1} a^{t-1}\left(\sum_{j=\left\lceil\frac{t-1}{2}\right\rceil}^{l} a^{-2 j} \sigma_{l-j}\left(b_{1}^{2}, \ldots, b_{l}^{2}\right)\left(\begin{array}{c}
2 j \\
t-1
\end{array}\right)\right) k^{t} \\
& +\sum_{t=0}^{2 l} a^{t-1}\left(\sum_{j=\left\lceil\frac{t}{2}\right\rceil}^{l} a^{-2 j} \sigma_{l-j}\left(b_{1}^{2}, \ldots, b_{l}^{2}\right)\left(\begin{array}{c}
2 j \\
t
\end{array}\right)\right) k^{t} \\
= & \sum_{t=0}^{2 l+1} a^{t-1}\left(\sum_{j=\left\lceil\frac{t-1}{2}\right\rceil}^{l} a^{-2 j} \sigma_{l-j}\left(b_{1}^{2}, \ldots, b_{l}^{2}\right)\left(\begin{array}{c}
2 j+1 \\
t
\end{array}\right)\right) k^{t} .
\end{aligned}
$$


Therefore, the desired inequality $\frac{\mathrm{g}_{t}(P)}{\mathrm{g}_{s}(P)} \leqslant a^{t-s} \frac{\left(\begin{array}{l}n \\ t\end{array}\right)}{\left(\begin{array}{l}n \\ s\end{array}\right)}$ is equivalent to

$$
\begin{aligned}
& \sum_{j=\left\lceil\frac{-1}{2}\right\rceil}^{l} a^{-2 j} \sigma_{l-j}\left(b_{1}^{2}, \ldots, b_{l}^{2}\right)\left(\begin{array}{c}
2 j+1 \\
t
\end{array}\right)\left(\begin{array}{c}
2 l+1 \\
s
\end{array}\right) \\
& \leqslant \sum_{j=\left\lceil\frac{s-1}{2}\right\rceil}^{l} a^{-2 j} \sigma_{l-j}\left(b_{1}^{2}, \ldots, b_{l}^{2}\right)\left(\begin{array}{c}
2 j+1 \\
s
\end{array}\right)\left(\begin{array}{c}
2 l+1 \\
t
\end{array}\right),
\end{aligned}
$$

and the further analysis is analogous to the even-dimensional case.

ii): This follows directly from part i), since

$$
a^{n} \mathrm{G}(P)=\sum_{s=0}^{n} a^{n} \mathrm{~g}_{s}(P) \geqslant \sum_{s=0}^{n}\left(\begin{array}{l}
n \\
s
\end{array}\right) a^{s} \mathrm{~g}_{n}(P)=(a+1)^{n} \operatorname{vol}(P) .
$$

The characterization of equality is inherited from part i) as well. prove

iii): Multiplying either side of the claimed inequality by $\frac{a^{n}}{\operatorname{vol}(P)}$, shows that we need to

$$
\frac{a^{n} \mathrm{G}(P)}{\operatorname{vol}(P)} \leqslant(a+1)^{n}-(a+1)^{n-2}+(a+1)^{n-2} \frac{a^{n}}{\operatorname{vol}(P)} .
$$

Again, we consider the case $n=2 l$ first. Using Equation (3.1) for $k=0$ and $k=1$ gives the equivalent inequality

$$
\prod_{j=1}^{l}\left((a+1)^{2}+\left(a b_{j}\right)^{2}\right) \leqslant(a+1)^{2 l}-(a+1)^{2 l-2}+(a+1)^{2 l-2} \prod_{j=1}^{l}\left(1+\left(a b_{j}\right)^{2}\right) .
$$

Expanding the products yields that this is equivalent to

$$
\sum_{j=0}^{l-1}(a+1)^{2 j} \sigma_{l-j}\left(\left(a b_{1}\right)^{2}, \ldots,\left(a b_{l}\right)^{2}\right) \leqslant \sum_{j=0}^{l-1}(a+1)^{2 l-2} \sigma_{l-j}\left(\left(a b_{1}\right)^{2}, \ldots,\left(a b_{l}\right)^{2}\right),
$$

which even holds summand-wise.

Equality holds if and only if $\sigma_{l-j}\left(\left(a b_{1}\right)^{2}, \ldots,\left(a b_{l}\right)^{2}\right)=0$, for all $j=0, \ldots, l-2$. Since $a>0$, this holds if and only if at most one of $b_{1}, \ldots, b_{l}$ is nonzero. This means, that there is at most one conjugate pair of roots of $\mathrm{G}(k P)$ having nonzero imaginary part.

The case $n=2 l+1$ is completely analogous.

Remark 3.2. The inequality in Theorem 3.1 iii) generalizes the linear inequality that is part of the characterization of 4-dimensional lattice polytopes having only roots with real part equal to $-\frac{1}{2}$ (see [5, Prop. 1.9]).

Applying Theorem 3.1 i) with $a=2$ and $s=0$ gives the following positive result concerning Wills' conjecture. 
Corollary 3.3. Conjecture 1.1 holds for every lattice polytope with the property that all the roots of its Ehrhart polynomial have real part equal to $-\frac{1}{2}$.

One may wonder whether lattice polytopes with an Ehrhart polynomial all of whose roots have real part equal to $-\frac{1}{a}$ actually exist. Let $P$ be a lattice polytope such that all roots of its Ehrhart polynomial have real part $-\frac{1}{2}$. Such polytopes are quite abundant, see [5]. Now, for every $l \in \mathbb{N}$, the roots of the Ehrhart polynomial of $l P$ have real part $-\frac{1}{2 l}$. This construction is not very satisfactory though.

Kasprzyk \& Nill [15] introduced the class of l-reflexive polytopes which contains more interesting examples with the desired property. Before we can give their definition, we need to fix some notation. A lattice point $u \in \mathbb{Z}^{n} \backslash\{0\}$ is primitive if the only lattice points contained in the line segment from 0 to $u$ are its endpoints. Every lattice polytope $P \in \mathcal{P}^{n}$ has a unique irredundant representation $P=\left\{x \in \mathbb{R}^{n}: u_{i}^{\top} x \leqslant l_{i}, i=1, \ldots, m\right\}$, where $u_{i} \in \mathbb{Z}^{n}$ are nonzero primitive lattice vectors and the $l_{i}$ are natural numbers. The index of $P$ is defined as the least common multiple of $l_{1}, \ldots, l_{m}$.

Definition 3.4 (l-reflexive polytope). A lattice polytope $P \in \mathcal{P}^{n}$ is called l-reflexive, for some $l \in \mathbb{N}$, if

i) the origin is contained in the interior of $P$,

ii) $l_{i}=l$, for all $i=1, \ldots, m$.

Note, that 1-reflexive polytopes are the much studied reflexive polytopes which have a close connection to algebraic geometry; see [18] and the references therein.

Remark 3.5. Kasprzyk \& Nill additionally require an l-reflexive polytope to have only primitive vertices, which discards multiples of reflexive polytopes. For our purposes it is more convenient to drop this assumption, and there should be no confusion here using the same name.

Now, Kasprzyk \& Nill study properties of $l$-reflexive polytopes and give a classification algorithm for the planar case. For example, they find $3605 l$-reflexive polygons with only primitive vertices and an index of at most 60 . The relation to our question is $[15$, Prop. 17] which says that every $l$-reflexive polygon with only primitive vertices, that is not unimodularly equivalent to the triangle with vertices $(-1,-1),(-1,2)$ and $(2,-1)$, has an Ehrhart polynomial all of whose roots have real part equal to $-\frac{1}{2 l}$.

We conclude by extending characterizations given in $[5,15]$. Recall that the polar of a polytope $P \in \mathcal{P}^{n}$ is defined as $P^{\star}=\left\{x \in \mathbb{R}^{n}: x^{\top} y \leqslant 1\right.$, for all $\left.y \in P\right\}$.

Proposition 3.6. Let $P \in \mathcal{P}^{n}$ be a lattice polytope with index $l$ that contains the origin in its interior. Then, the following are equivalent:

i) $P$ is an l-reflexive polytope,

ii) $l P^{\star}$ is a lattice polytope all of whose vertices are primitive,

iii) $\mathrm{g}_{n-1}(P)=\frac{n}{2 l} \operatorname{vol}(P)$. 
Proof. The equivalence of i) and ii) can be read off from the proof of [15, Prop. 2].

i) $\Longleftrightarrow$ iii): Let $F_{i}=\left\{x \in \mathbb{R}^{n}: u_{i}^{\top} x=l_{i}\right\} \cap P$, for $i=1, \ldots, m$, be the facets of $P$, where as before the $u_{i}$ are primitive normal vectors. As Ehrhart [9] already showed (cf. [2, Thm. 5.6])

$$
\mathrm{g}_{n-1}(P)=\frac{1}{2} \sum_{i=1}^{m} \frac{\operatorname{vol}_{n-1}\left(F_{i}\right)}{\operatorname{det}\left(\operatorname{aff} F_{i} \cap \mathbb{Z}^{n}\right)}
$$

Therein, $\operatorname{vol}_{n-1}\left(F_{i}\right)$ denotes the $(n-1)$-dimensional volume of the facet $F_{i}$, aff $F_{i}$ its affine hull, and $\operatorname{det}\left(\right.$ aff $\left.F_{i} \cap \mathbb{Z}^{n}\right)=\left\|u_{i}\right\|$ (see [16, Prop. 1.2.9]) the determinant of the sublattice of $\mathbb{Z}^{n}$ contained in aff $F_{i}$.

Now, if $P$ is $l$-reflexive, then $l_{i}=l$ for all $i=1, \ldots, m$, and writing $F_{i}^{o}=\operatorname{conv}\left\{0, F_{i}\right\}$, we have

$$
\begin{aligned}
\mathrm{g}_{n-1}(P) & =\frac{1}{2} \sum_{i=1}^{m} \frac{\operatorname{vol}_{n-1}\left(F_{i}\right)}{\operatorname{det}\left(\operatorname{aff} F \cap \mathbb{Z}^{n}\right)}=\frac{n}{2 l} \sum_{i=1}^{m} \frac{l \cdot \operatorname{vol}_{n-1}\left(F_{i}\right)}{n \cdot\left\|u_{i}\right\|} \\
& =\frac{n}{2 l} \sum_{i=1}^{m} \operatorname{vol}\left(F_{i}^{o}\right)=\frac{n}{2 l} \operatorname{vol}(P) .
\end{aligned}
$$

Conversely, let $\mathrm{g}_{n-1}(P)=\frac{n}{2 l} \operatorname{vol}(P)$. Similarly as above, we get

$$
\sum_{i=1}^{m} \frac{l_{i} \cdot \operatorname{vol}_{n-1}\left(F_{i}\right)}{n \cdot\left\|u_{i}\right\|}=\operatorname{vol}(P)=\frac{2 l}{n} \mathrm{~g}_{n-1}(P)=\sum_{i=1}^{m} \frac{l \cdot \operatorname{vol}_{n-1}\left(F_{i}\right)}{n \cdot\left\|u_{i}\right\|}
$$

Therefore, $\sum_{i=1}^{m}\left(l-l_{i}\right) \frac{\operatorname{vol}_{n-1}\left(F_{i}\right)}{\left\|u_{i}\right\|}=0$. Since $l \geqslant l_{i}$, for all $i=1, \ldots, m$, we get $l=l_{i}$, for all $i=1, \ldots, m$, hence $P$ is $l$-reflexive.

Bey, Henk \& Wills [5, Prop. 1.8] showed that every lattice polytope $P \in \mathcal{P}^{n}$ whose Ehrhart polynomial has only roots with real part equal to $-\frac{1}{2}$ is unimodularly equivalent to a reflexive polytope. Their arguments apply in order to extend [15, Prop. 16] as follows:

Corollary 3.7. Let $P \in \mathcal{P}^{n}$ be a lattice polytope with index $l$ that contains the origin in its interior. If all roots of the Ehrhart polynomial of $P$ have real part equal to $-\frac{1}{2 l}$, then, up to a unimodular transformation, $P$ is an l-reflexive polytope.

Proof. Recall that $-\gamma_{1}(P), \ldots,-\gamma_{n}(P)$ are the roots of the Ehrhart polynomial of $P$. It is not hard to see that $\frac{\mathrm{g}_{n-1}(P)}{\operatorname{vol}(P)}=\sum_{i=1}^{n} \gamma_{i}(P)$. Since the roots come in conjugate pairs, we get $g_{n-1}(P)=\frac{n}{2 l} \operatorname{vol}(P)$ and Proposition 3.6 iii) shows that $P$ is $l$-reflexive.

We close with an open question.

Problem 3.8. Do there exist lattice polytopes whose Ehrhart polynomial has only roots with real part equal to $-\frac{1}{a}$, for some $a \notin 2 \mathbb{N}$ ? 


\section{Acknowledgements}

We thank Martin Henk for fruitful discussions on the topic and very useful remarks on an earlier draft. Moreover, we are grateful to the referee for pointers to the literature and suggestions on the presentation.

\section{References}

[1] Milton Abramowitz and Irene A. Stegun, editors. Handbook of mathematical functions with formulas, graphs, and mathematical tables. Dover Publications Inc., New York, 1992. Reprint of the 1972 edition.

[2] Matthias Beck and Sinai Robins. Computing the continuous discretely. Undergraduate Texts in Mathematics. Springer, New York, 2007. Integer-point enumeration in polyhedra.

[3] Ulrich Betke, Martin Henk, and Jörg M. Wills. Successive-minima-type inequalities. Discrete Comput. Geom., 9(2):165-175, 1993.

[4] Christian Bey, Martin Henk, Matthias Henze, and Eva Linke. Notes on lattice points of zonotopes and lattice-face polytopes. Discrete Mathematics, 311(8-9):634-644, 2011.

[5] Christian Bey, Martin Henk, and Jörg M. Wills. Notes on the roots of Ehrhart polynomials. Discrete Comput. Geom., 38(1):81-98, 2007.

[6] Benjamin Braun. An Ehrhart series formula for reflexive polytopes. Electron. J. Combin., 13(1):Note 15, 5 pp. (electronic), 2006.

[7] Benjamin Braun. Norm bounds for Ehrhart polynomial roots. Discrete Comput. Geom., 39(1-3):191-193, 2008.

[8] Jesús A. De Loera, Brandon Dutra, Matthias Köppe, Stanislav Moreinis, Gregory Pinto, and Jianqiu Wu. A user's guide for latte integrale v1.5. software package LattE is available at http://www.math.ucdavis.edu/ latte/, 2011.

[9] Eugène Ehrhart. Sur un problème de géométrie diophantienne linéaire II. J. Reine Angew. Math., 227:25-49, 1967.

[10] Eugène Ehrhart. Sur la loi de réciprocité des polyèdres rationnels. Comptes Rendus de l'Académie des Sciences Paris, Séries A-B, 266:A696-A697, 1968.

[11] Ewgenij Gawrilow and Michael Joswig. polymake: a Framework for Analyzing Convex Polytopes. In Gil Kalai and Günter M. Ziegler, editors, Polytopes - Combinatorics and Computation, pages 43-74. Birkhäuser, 2000.

[12] Peter Gritzmann and Jörg M. Wills. Lattice points. In Handbook of convex geometry, Vol. B, pages 765-797. North-Holland, Amsterdam, 1993.

[13] Christian Haase and Tyrrell B. McAllister. Quasi-period collapse and $\mathrm{GL}_{n}(\mathbb{Z})$-scissors congruence in rational polytopes. In Integer points in polyhedra-geometry, number 
theory, representation theory, algebra, optimization, statistics, volume 452 of Contemp. Math., pages 115-122. American Mathematical Society, Providence, RI, 2008.

[14] Olof Hanner. Intersections of translates of convex bodies. Mathematica Scandinavica, 4:67-89, 1956.

[15] Alexander M. Kasprzyk and Benjamin Nill. Reflexive polytopes of higher index and the number 12. Electron. J. Combin., 19(3):P9, 2012.

[16] Jacques Martinet. Perfect lattices in Euclidean spaces, volume 327 of Grundlehren der Mathematischen Wissenschaften [Fundamental Principles of Mathematical Sciences]. Springer-Verlag, Berlin, 2003.

[17] Hermann Minkowski. Geometrie der Zahlen. Bibliotheca Mathematica Teubneriana. Teubner, Leipzig-Berlin, 1896. reprinted by Johnson Reprint Corp., New York, 1968.

[18] Benjamin Nill. Gorenstein toric Fano varieties. PhD thesis, University of Tübingen, 2005. http://w210.ub.uni-tuebingen.de/dbt/volltexte/2005/1888/pdf / nill.pdf.

[19] Jörg M. Wills. On an analog to Minkowski's lattice point theorem. In The geometric vein, pages 285-288. Springer, New York, 1981. 